\title{
Como avaliar o modo como as pessoas se percebem fisicamente? Um olhar sobre a versão portuguesa do Physical Self-Perception Profile (PSPP)
}

\author{
António Manuel Fonseca \\ Faculdade de Ciências do Desporto e de Educação Física \\ Universidade do Porto \\ Portugal
}

\author{
Kenneth Fox \\ Universidade de Bristol \\ Inglaterra
}

\section{RESUMO}

O objectivo desta pesquisa consistiu na análise das propriedades psicométricas da versão portuguesa do Physical Self-Perception Profile (PSPP): o PSPPp. Participaram neste estudo 729 adultos (idades compreendidas entre os 20 e os 50 anos; média de $31.18 \pm 8.27$ anos) de ambos os sexos (470 do sexo feminino e 259 do sexo masculino), que praticavam uma actividade física ou desportiva de forma não competitiva. Os dados foram divididos em duas metades equivalentes. A primeira foi submetida a uma Análise Factorial Exploratória que permitiu identificar duas soluções constituídas por 3 e 4 factores. Em seguida, utilizando a outra metade dos dados, foi realizada uma Análise Factorial Confirmatória a quatro modelos distintos: i) M424, constituído por 24 itens distribuídos por 4 factores (idêntico ao da versão original do PSPP: Competência Desportiva, Condição Física, Aparência Física e Força Física); ii) M324, com 24 itens e 3 factores (considerando os dois primeiros factores conjuntamente); iii) M416, com 16 itens e 4 factores (similar ao da versão original mas com apenas 4 itens por factor); e iv) M312, com 12 itens e 3 factores (similar ao modelo 2, mas com apenas 4 itens por factor). A análise dos vários indicadores de ajustamento global seleccionados forneceu evidência clara no sentido da bondade do ajustamento do modelo M312 e da sua superioridade relativamente aos outros modelos, razão pela qual se recomenda a sua utilização. Ainda assim, é recomendada a realização de mais trabalho no sentido do completo estabelecimento da validade e fiabilidade do PSPPp, bem como do desenvolvimento de uma escala destinada a avaliar a capacidade física funcional percebida pelos indivíduos.

Palavras-chave: autopercepções físicas, avaliação, instrumento, propriedades psicométricas.

\author{
ABSTRACT \\ How do people perceive themselves physically? \\ A look into the Portuguese version of the Physical \\ Self-Perception Profile (PSPP).
}

The purpose of this research was to analyse the psychometric properties of the Portuguese version of Physical Self-Perception Profile (PSPP): PSPP. The sample consisted of 729 adults aged between 20-50 years $(31.18 \pm 8.27$ years) of both sexes (470 females and 259 males) who participated regularly in physical activities or recreational sports. Data were divided in two equivalent halves. The first half was analysed with Exploratory Factor Analysis that identified two solutions with 3 and 4 factors. The second half of the data was used to test four different models by Confirmatory Factor Analysis: i) M424, with 24 items and 4 factors (identical to the original version of PSPP: Sport Competence, Physical Condition, Body Appearance and Physical Strength); ii) M324, with 24 items and 3 factors (considering first two factors jointly); iii) M416, with 16 items and 4 factors (similar to original version but with only 4 items for factor); and iv) M312, with 12 items and 3 factors (similar to model 2, but with only 4 items for factor). Analysis of several standard criteria showed that the model M312 showed the better fit to the data, and therefore its use is recommended. Nevertheless, further work is needed in order to establish the validity and reliability of PSPPp, as well as the development of a scale to evaluate the perceived functional physical capacity in adults.

Keywords: physical self-perceptions, assessment, instrument, psychometric properties. 


\section{INTRODUÇÃO}

Apesar de parecer ser consensual associar à prática regular de actividade física diversos benefícios de natureza psicológica $(9,34,35)$, a investigação no domínio das auto-percepções físicas pode considerar-se ainda como insuficiente (16).

Até há relativamente pouco tempo, uma das principais limitações ao desenvolvimento da investigação neste domínio consistia na escassez de instrumentos fiáveis e válidos para proceder à caracterização e avaliação do modo como os indivíduos percebem a sua dimensão física. No entanto, nos últimos anos foram desenvolvidos alguns instrumentos que têm vindo a revelar-se como fiáveis e válidos para esse fim, contribuindo, desse modo, para o desenvolvimento do conhecimento neste domínio.

De entre estes, um dos que mais se tem destacado positivamente é o Physical Self-Perception Profile (PSPP) (18). O PSPP, cujos autores se basearam em grande medida nos trabalhos de Susan Harter, possibilita aos inquiridos a indicação das suas percepções relativamente a si próprios no que concerne a vários aspectos do seu 'eu físico'.

O PSPP é constituído por cinco escalas: competência desportiva (i.e., percepções acerca da capacidade desportiva e atlética, capacidade para aprender técnicas desportivas, e confiança em contextos desportivos), condição física (i.e., percepções acerca do nível de condição física, estamina e fitness, capacidade para persistir na prática de actividade física, e confiança em contextos de actividade física e fitness), aparência física (i.e., percepções acerca da atracção exercida nos outros pela sua figura ou físico, capacidade para manter um corpo atraente, e confiança na aparência), força física (i.e., percepções acerca da força físi$\mathrm{ca}$, desenvolvimento muscular, e confiança em situações que exigem força) e auto-estima física (i.e., sentimentos generalizados de alegria, satisfação, orgulho, respeito e confiança no 'eu físico').

A inclusão da escala relativa à auto-estima física procurou permitir a avaliação de dois níveis distintos de autopercepção física, ordenados verticalmente em termos de especificidade (situacional/genérico). Assim, enquanto as quatro primeiras escalas permitem recolher informação acerca do modo como os indivíduos se percepcionam relativamente a cada uma das quatro facetas anteriormente descritas, a escala da auto-estima física orienta-se para a recolha de informação referente ao modo como eles se sentem em relação à sua dimensão física em geral. Cada uma das cinco escalas do PSPP é igualmente constituída por seis itens, relativamente a cada um dos quais os inquiridos se pronunciam através de uma estrutura de resposta igual à proposta por Harter; isto é, de 'formato de estrutura alternativa'. Neste formato, cada item consiste num par de afirmações contrárias (e.g., «algumas pessoas sentem que não são muito boas quando se trata de praticar desporto" mas "outras sentem que são realmente boas acerca de qualquer desporto»), devendo o inquirido, numa primeira fase, seleccionar a afirmação que lhe parece descrevê-lo melhor, para, em seguida, indicar o grau de semelhança que entende que essa afirmação tem consigo (i.e., se entende que, no seu caso, a afirmação seleccionada é «realmente verdade», ou apenas «mais ou menos verdade»).

A opção por este formato - que normalmente provoca uma maior dificuldade aos inquiridos para responder às questões $(15,25,36)$ - baseou-se no facto de ter vindo a ser demonstrado que deste modo é eliminada a tendência dos indivíduos para responderem de acordo com o que consideram ser socialmente mais aceite ou desejável $(15,17,18)$.

O PSPP, ao permitir a avaliação simultânea de diferentes facetas do eu físico (18), tem sido por vezes utilizado em combinação com um instrumento de avaliação da auto-estima global, como por exemplo o Inventário de Auto-Estima de Rosenberg (33), fornecendo, dessa forma, uma estrutura conceptual útil para a investigação dos mecanismos de modificação da auto-estima através da actividade física ou desportiva.

Inclusivamente, Sonstroem, Harlow e Josephs (37) modificaram o modelo anteriormente proposto por Sonstroem e Morgan (38) exactamente com esse objectivo, considerando a existência de dois níveis diferenciados de competência física percebida, tendo verificado que os dados recolhidos no âmbito de um estudo realizado com uma amostra de praticantes de aeróbica do sexo feminino suportaram essa alteração. Mais tarde, também Whitehead (45), na sequência de um estudo realizado com mais de 500 estudantes, declarou ter encontrado suporte para a hipótese da auto-estima física se situar a um nível intermédio entre a auto-estima geral e as quatro facetas repre- 
sentadas pelas escalas do PSPP, neste caso de uma versão adaptada para crianças.

O PSPP tem sido utilizado em numerosos estudos e a sua fiabilidade e validade na avaliação das auto-percepções dos indivíduos tem sido salientada por diversas vezes $(15,18,25,31,39)$. Aliás, a qualidade deste instrumento pode ser aferida, por exemplo, a partir das palavras de dois conceituados autores neste domínio: Sonstroem (36) - «O desenvolvimento do Physical Self-Perception Profile (PSPP) (Fox \& Corbin, 1989) representa um importante avanço no estudo do eu físico (p.11)» - e Marsh (23) - «O PSPP é o instrumento multidimensional do autoconceito físico mais robusto do directório de Ostrow (1990 (p.40)" [directório de testes psicológicos relacionados com as ciências do desporto e do exercício]. De recordar, porém, que o PSPP foi desenvolvido originalmente nos Estados Unidos da América com jovens estudantes universitários, tendo Fox (15) recomendado que a sua aplicação a outras populações deveria ser acompanhada pela realização de uma extensa análise às suas propriedades psicométricas, no sentido da determinação da sua validade e fiabilidade. Mais recentemente, também Marsh (23) secundou as recomendações de Fox, reforçando a necessidade de realizar análises psicométricas extensivas ao PSPP aquando da sua utilização em situações e populações distintas daquelas com as quais foi inicialmente desenvolvido.

Desde a sua publicação, o PSPP foi rapidamente adoptado por investigadores de diversos países, ao ponto de estar hoje disponível em 9 línguas diferentes, entre as quais a portuguesa. De facto, o PSPP foi traduzido para a língua portuguesa no âmbito de uma cooperação iniciada em 1995 sob a coordenação dos autores deste estudo (13), e utilizado posteriormente nalguns estudos desenvolvidos entre nós $(14,29,30)$.

Para que a versão traduzida fosse o mais possível equivalente à versão original, quer do ponto de vista semântico quer do conteúdo, baseámo-nos nas sugestões de Vallerand (42) relativamente à tradução e adaptação transcultural de instrumentos psicológicos. Assim, a tradução inicial do PSPP para a língua portuguesa foi realizada por dois especialistas bilingues, tendo essa tradução sido, posteriormente, sujeita à apreciação de um júri, constituído por psicólogos, treinadores e tradutores. Concluída esta fase, foram realizadas entrevistas com atletas com características diferentes entre si (e.g., sexo, idade, modalidade desportiva praticada), no sentido de determinar a compreensibilidade e uniformidade intercontextual do instrumento. Atendendo a que não foram identificadas quaisquer dificuldades por parte dos inquiridos na resposta aos seus diversos itens, considerámos criada a versão portuguesa do PSPP, por nós denominada de Perfil de Auto-Percepção Física (PSPPp) (12).

Para a realização dos estudos anteriormente referidos, foi naturalmente desenvolvido um processo de avaliação da fiabilidade e validade do PSPPp, tendo os resultados sido prometedores. Todavia, em nenhum desses estudos, se recorreu, por exemplo, à estatística multivariada da análise factorial, actualmente considerada como imprescindível para que se possa concluir efectivamente no sentido da validade e fiabilidade de um instrumento psicológico, principal razão pela qual decidimos desenvolver o presente estudo. Efectivamente, parece constituir-se hoje como inequívoca a utilidade, ou mesmo necessidade, de, no domínio da análise da validade de um instrumento psicológico, se recorrer à análise factorial exploratória (AFE) ou confirmatória (AFC), decorrendo a opção entre elas da existência ou não de uma ideia prévia relativamente consistente sobre a estrutura mais adequada ao instrumento em questão.

Enquanto a AFE é utilizada para explorar um conjunto de dados, e determinar o número e natureza dos factores que contribuem para a covariância entre as variáveis investigadas, quando o investigador não possui ainda suficientes evidências no sentido da elaboração de uma hipótese relativamente aos factores que lhes estão subjacentes, a AFC é utilizada quando antecipadamente é possível elaborar essa hipótese com alguma segurança.

Daí que se refira frequentemente que, enquanto com a AFE se procura construir teoria, com a AFC procura-se testar essa mesma teoria (40), resultando portanto que a AFE seja mais associada à fase de desenvolvimento de um instrumento e a AFC à fase de determinação da sua validade.

Em decorrência do anteriormente exposto, e atendendo a que o objectivo do nosso estudo passava pelo exame às propriedades psicométricas de um instrumento já existente, designadamente no que 
concerne à determinação da validade da sua estrutura factorial, seria natural que a nossa opção fosse no sentido da utilização exclusiva da AFC.

Todavia, nem sempre a análise dos resultados recolhidos através da aplicação de versões traduzidas do PSPP para outras línguas e culturas tem permitido identificar sem problemas a estrutura proposta para a versão original do instrumento, especificamente no que se reporta ao número de factores que a constituem $(19,20,43)$.

Logo, em lugar de procurar avaliar imediatamente, através da AFC, o grau de correspondência entre a estrutura proposta para a versão original e os dados relativos à nossa realidade, entendemos ser mais correcto desenvolver um estudo inicial no sentido de recolher o máximo possível de informação relativamente a formas alternativas de agrupamento das diferentes variáveis que constituem o PSPPp, utilizando para o efeito a AFE, e apenas depois procurar testar a sua validade, a partir do recurso à AFC.

\section{MATERIAL E MÉTODOS}

\section{Amostra e procedimentos}

Participaram neste estudo 729 adultos de ambos os sexos (470 do sexo feminino e 259 do sexo masculino), praticantes de jogging, aeróbica, natação e musculação, em contextos não competitivos, com idades compreendidas entre os 20 e os 50 anos (média de $31.18 \pm 8.27$ anos). O preenchimento do PSPPp foi efectuado antes ou após uma das suas aulas e decorreu sem dificuldades.

\section{Perspectiva global sobre a análise dos dados}

Para a inspecção à estrutura factorial do PSPPp, apenas foram considerados 24 dos seus 30 itens, tendo sido excluídos os constituintes da escala da auto-estima física. Esta decisão decorreu da consideração de que, em concordância com a teoria que esteve subjacente à elaboração do PSPP, a escala relativa à auto-estima física não deve ser colocada a um mesmo nível de análise, porquanto corresponde a um contructo super-ordenador resultante dos outros quatro constructos (18).

Tal como referimos anteriormente, a nossa investigação desenvolveu-se em duas fases fundamentais: a primeira, essencialmente exploratória, e a segunda confirmatória. $\mathrm{Na}$ linha do proposto nesta área do conhecimento, dividimos aleatoriamente os dados relativos à amostra total em duas metades equivalentes, utilizando uma delas para realizar o estudo exploratório e a outra para o estudo confirmatório. Pedhazur e Schmelkin (32) alertaram para o facto de que "a análise factorial exploratória não é, ou não deve ser, um processo no qual todo o tipo de variáveis é introduzido numa 'trituradora' análise factorial, na esperança de que dela emirja algo com significado (p.591)".

Nessa medida, e atendendo igualmente a que, tal como alertou Mulaik (27), "racionalmente, não existe forma óptima de extrair conhecimento da experiência sem efectuar determinadas assunções prévias (p.265)", decidimos condicionar o processo de extracção dos factores, definindo que eles deveriam ser três ou quatro. Para esta opção, concorreu, fundamentalmente, o reconhecimento de que, por um lado, a estrutura factorial original do PSPP é constituída por quatro factores, mas, por outro, por em alguns dos estudos desenvolvidos com versões traduzidas do PSPP apenas terem sido identificados três factores.

Quanto ao método a seguir para realizar a rotação dos factores, e apesar de ser hábito os investigadores optarem pela rotação varimax, baseámo-nos uma vez mais nas indicações da literatura, designadamente no que se refere ao facto de a teoria subjacente à construção do PSPP postular que os factores que o constituem se correlacionam uns com os outros, optando pela rotação oblimin.

Para a retenção de um item num factor, decidimos considerar apenas os que apresentassem um valor de saturação igual ou superior a .40 nesse factor, correspondente à partilha entre eles de, pelo menos, $10 \%$ da variância (41).

Com a segunda fase da nossa investigação, procurámos determinar qual o modelo de medida mais ajustado à matriz de covariância do segundo conjunto de dados, analisando em alternativa a estrutura factorial proposta para a versão original e a estrutura identificada durante a primeira fase do estudo.

Adicionalmente, considerámos importante tentar verificar se seria possível diminuir o número original de seis itens por factor, sem que tal redução exercesse um impacte negativo ao nível das suas propriedades psicométricas, porquanto tal parcimónia permiti- 
ria tornar mais fácil e rápida a aplicação do PSPPp, e isso se constituiria como uma vantagem a considerar pelos investigadores.

De uma forma geral, considera-se que dois indicadores por factor é o número mínimo, sendo todavia mais seguro optar por três ou quatro. Aliás, caso as correlações entre os factores sejam elevadas, o mais aconselhável é optar por quatro indicadores por factor, entendendo-se, normalmente, que cinco ou mais indicadores por factor é mais do que suficiente.

Assim sendo, e considerando que, pelo menos no plano teórico, os vários factores do PSPPp se correlacionam entre si, decidimos optar por testar alternativamente uma solução com apenas quatro indicadores por factor.

Para determinar se um modelo de medida apresenta ou não um bom grau de correspondência com um conjunto de dados existe actualmente uma elevada profusão de índices (para mais detalhes sobre índices de bondade de ajustamento de um modelo de medida, ver $[3,8,24,26])$. Nessa medida, decidimos optar apenas pelos que parecem reunir o maior consenso, sendo por isso utilizados pela maior parte dos investigadores no domínio da análise da validade de instrumentos psicológicos.

Um dos índices ao qual se recorre imediatamente é o $\chi^{2}$, o qual testa a hipótese de que o modelo de medida proposto é consistente com a matriz de covariância dos dados examinados: quanto mais baixo o seu valor, maior a consistência entre eles.
No entanto, como este índice é reconhecidamente sensível à dimensão da amostra (essencialmente, a partir de um $n>200$ ), os investigadores desenvolveram outros índices para avaliar a qualidade global do ajustamento dos modelos, tais como o goodness of fit index (GFI) e o adjusted goodness of fit index (AGFI), gerados pelo programa estatístico LISREL. Outros índices também considerados frequentemente pelos investigadores são o rácio entre o $\chi^{2}$ e os graus de liberdade, o comparative of fit index (CFI), a standardized root mean square residual (RMRst) e a root mean square of error of aproximation (RMSEA). No nosso estudo, decidimos considerar todos os índices anteriormente referidos.

\section{RESULTADOS}

$A$ inspecção às duas soluções identificadas a partir da AFE (ver quadro 1) revelou que quase não se verificaram casos de saturação cruzada, já que, com uma ou outra excepção, cada item saturou de forma evidente apenas num dos factores (as excepções foram os itens 02 e 08 na solução de 4 factores, e os itens 02 , 08 e 28 na solução de 3 factores: todos eles saturaram em dois factores). No que concerne à proporção da variância explicada por cada uma delas, verificámos que enquanto a solução de três factores explicava quase $50 \%$ da variância das respostas aos itens, a de quatro factores explicava aproximadamente $5 \%$ mais. 
Quadro 1. Análise Factorial Exploratória [rotação oblimin] ao PSPPp. Soluções com três e quatro factores.

\begin{tabular}{|c|c|c|c|c|c|c|c|}
\hline & \multicolumn{4}{|c|}{ AFE - 4 FACTORES } & \multicolumn{3}{|c|}{ AFE - 3 FACTORES } \\
\hline & Competência & Aparência & Força & Condição & Confiança & Aparência & Força \\
\hline & Desportiva & Física & Física & Física & Física & Física & Física \\
\hline PSPPp.24 & & & 77 & & & & .70 \\
\hline PSPPp.04 & & & .78 & & & & .78 \\
\hline PSPPp.14 & & & .64 & & & & .64 \\
\hline PSPPp.09 & & & .54 & & & & .63 \\
\hline PSPPp.19 & & & .46 & & & & .46 \\
\hline PSPPp.29 & & & .70 & & & & .55 \\
\hline PSPPp.06 & & .49 & & & & .51 & \\
\hline PSPPp.16 & & & & & .48 & & \\
\hline PSPPp.17 & .75 & & & & .75 & & \\
\hline PSPPp.13 & .61 & & & & .66 & & \\
\hline PSPPp.11 & .59 & & & & .68 & & \\
\hline PSPPp.21 & .55 & & & & .59 & & \\
\hline PSPPp.01 & .62 & & & & .66 & & \\
\hline PSPPp.02 & .47 & .42 & & & .44 & .51 & \\
\hline PSPPp.03 & & .76 & & & & .71 & \\
\hline PSPPp.18 & & 77 & & & & .75 & \\
\hline PSPPp.08 & .44 & .49 & & & .43 & .53 & \\
\hline PSPPp.28 & & .46 & & & .40 & .41 & \\
\hline PSPPp.23 & & & .47 & & & & \\
\hline PSPPp.07 & & & & .72 & & & .52 \\
\hline PSPPp.22 & & & & .65 & .41 & & \\
\hline PSPPp.12 & .41 & & & & .51 & & \\
\hline PSPPp.27 & & & .44 & & & & .51 \\
\hline PSPPp.26 & & & & .60 & .41 & & \\
\hline Valor próprio & 8.47 & 1.73 & 1.45 & 1.31 & 8.47 & 1.73 & 1.45 \\
\hline Variância explicada & 35.28 & 7.18 & 6.06 & 5.47 & 35.28 & 7.18 & 6.06 \\
\hline
\end{tabular}

Ao compararmos as duas soluções, verificámos que os seus segundo e terceiro factores eram praticamente idênticos (com uma única excepção: enquanto na solução de quatro factores aos itens $04,09,14,19$, 24,27 e 29 se associou o item 23 , na solução de três factores, em lugar do item 23 , foi o item 07 que se associou aos itens anteriormente indicados), e que o primeiro factor da solução de três factores integrou praticamente todos os itens que constituíam o primeiro e o quarto factores da solução de quatro factores. Efectivamente, da análise à constituição de cada um dos factores, resultou que os segundo e terceiro factores de ambas as soluções se assemelharam em grande medida, e respectivamente, aos factores
Aparência Física e Força Física da estrutura original do PSPP, razão porque decidimos manter aquelas designações. Seguindo o mesmo raciocínio, e no que concerne à solução de quatro factores, decidimos manter igualmente as designações dos outros dois factores da estrutura original do PSPP, ainda que no caso do quarto factor, designado por Condição Física, essa semelhança não tivesse sido tão evidente, fundamentalmente porque foi constituído por apenas três itens.

Relativamente à solução de três factores, decidimos denominar o primeiro factor de Confiança Física, não só pelo facto de nele terem saturado a maioria dos itens dos factores originais Competência 
Desportiva e Condição Física, mas também pelo facto de os itens que nele saturaram de forma mais evidente parecerem sugerir acima de tudo a existência de uma variável mais relacionada com o à-vontade manifestado pelas pessoas em situações de actividade física ou desportiva; ou seja, este factor, mais do que apontar no sentido da obtenção de elevados níveis de rendimento, parece indiciar a existência de uma elevada confiança por parte das pessoas na participação em actividades daquela natureza.

Considerando os resultados da AFE, decidimos realizar AFC's para comparar o grau de ajustamento do modelo de três factores decorrente da AFE, bem como o do modelo original do PSPP, à segunda metade dos dados recolhidos. Para além disso, decidimos ainda investigar o grau de ajustamento de outros dois modelos de medida, globalmente semelhantes aos dois anteriormente referidos, mas constituídos por um menor número de itens; mais especificamente, quatro por factor.

Assim sendo, foram submetidos à AFC quatro modelos de medida distintos do PSPPp:

i) um modelo $\mathrm{M}_{24}^{4}$ (ver Figura 1), baseado nas sugestões dos autores da versão original do PSPP, com os 24 itens distribuídos por quatro factores: Competência Desportiva (itens 01, 06, 11, 16, 21, e 26) Condição Física $(02,07,12,17,22$, e 27), Aparência Física $(03,08,13,18,23$, e 28$)$ e Força Física $(04,09,14,19,24$, e 29);

ii) um modelo $\mathrm{M}^{3}{ }_{24}$ (ver Figura 2), baseado nos resultados da AFE anteriormente realizada, com os 24 itens distribuídos por três factores: Confiança Física (itens 01, 02, 06, 07, 11, 12, $16,17,21,22,26$ e 27), Aparência Física (03, 08, 13, 18, 23, e 28) e Força Física $(04,09,14$, 19, 24, e 29);

iii) um modelo $\mathrm{M}_{16}^{4}$ (ver Figura 3), baseado nas sugestões dos autores da versão original do PSPP, com os 16 itens distribuídos por quatro factores: Competência Desportiva (itens 01, 11, 16, e 21) Condição Física (07, 12, 22, e 27), Aparência Física $(03,08,18$, e 28) e Força Física (04, 09, 14 , e 24). iv) um modelo $\mathrm{M}^{3}{ }_{12}$ (ver Figura 4), baseado nos resultados da AFE anteriormente realizada, com os 12 itens distribuídos por três factores: Confiança Física (itens 01, 11, 17, e 21), Aparência Física $(03,08,18$, e 28) e Força Física $(04,09,14$, e 24$)$.

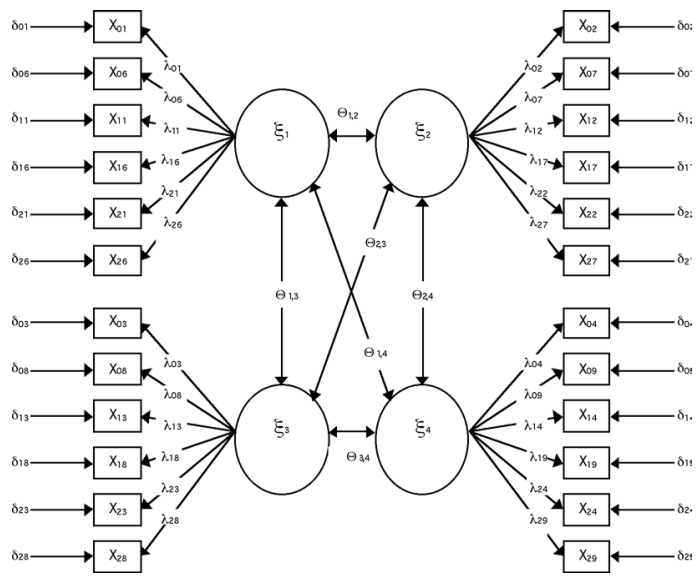

Figura 1. Modelo de medida $\mathrm{M}_{24}^{4}$ do PSPPp.

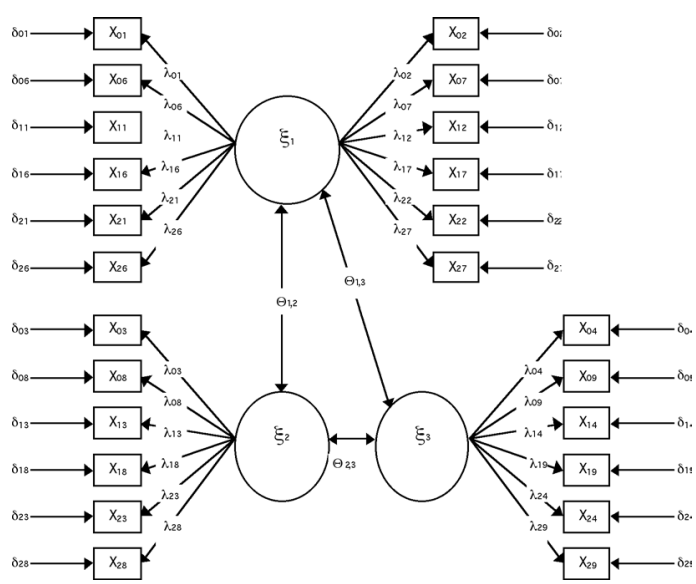

Figura 2. Modelo de medida $M^{3}{ }_{24}$ do PSPPp. 


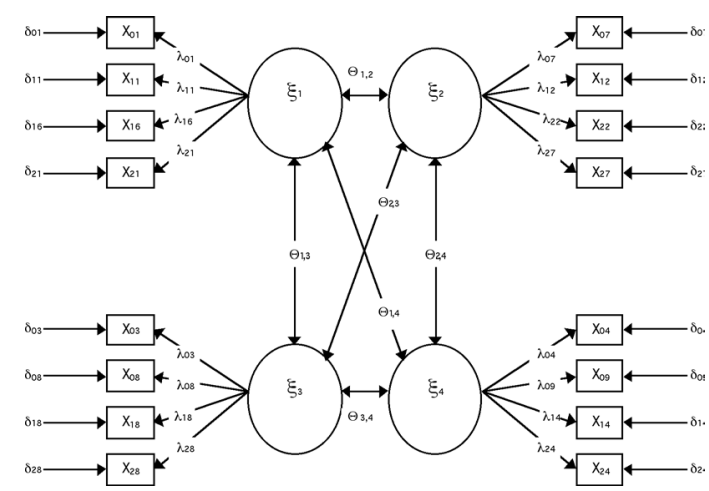

Figura 3. Modelo de medida $\mathrm{M}_{16}^{4}$ do PSPPp.

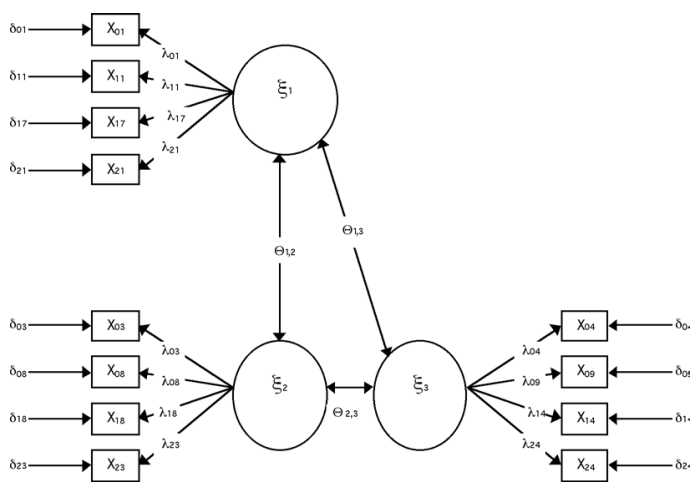

Figura 4. Modelo de medida $M_{12}^{3}$ do PSPPp.

Da análise comparativa dos valores dos indicadores correspondentes à bondade do ajustamento global de cada um dos modelos de medida submetidos à AFC (ver quadro 2) resultou claramente a superioridade do modelo M312, relativamente a todos os outros.

Quadro 2. Índices de bondade do ajustamento global para cada um dos modelos inspeccionados.

\begin{tabular}{lcccccccc} 
Modelo & $\chi^{1}$ & gl & $\chi^{1 / g l}$ & GFI & AGFI & CFI & RMSEA RMRst \\
\hline M424 & $733.37 p<.001$ & 246 & 2.98 & .82 & .78 & .85 & .089 & .064 \\
M324 & $1011.25 p<.001$ & 249 & 4.06 & .81 & .77 & .73 & .094 & .085 \\
M416 & $418.72 p<.001$ & 98 & 4.27 & .88 & .84 & .81 & .089 & .070 \\
M312 & $110.14 p<.001$ & 50 & 2.20 & .96 & .93 & .94 & .051 & .051
\end{tabular}

De facto, enquanto o modelo $\mathrm{M}^{3}{ }_{12}$, apesar do já esperado valor significativo do $\chi^{1}$ (atendendo à dimensão da amostra), apresentou valores perfeitamente aceitáveis em todos os outros indicadores, reflectindo assim um bom ajustamento aos dados examinados, o mesmo não se verificou para os outros modelos inspeccionados.

Na verdade, ainda que não exista um consenso absoluto relativamente aos valores a partir dos quais deve considerar-se que um modelo de medida se ajusta de forma aceitável a um determinado conjunto de dados, a maior parte dos especialistas concorda que o valor de corte a observar, para os indicadores relativos à sua bondade de ajustamento global seleccionados para este estudo (i.e., GFI, AGFI, CFI), deve estabelecer-se em .90: valores iguais ou superiores a .90 reflectem que o modelo em causa se ajusta de forma aceitável à matriz de covariância inspeccionada, valores inferiores significam o contrário (para mais detalhes, ver por exemplo [8]).

Considerando a reconhecida dependência da estatística do $\chi^{1}$ à dimensão da amostra, é frequente os investigadores assumirem a rácio entre o $\chi^{1}$ e os graus de liberdade como um indicador ad hoc do ajustamento do modelo de medida, conquanto não haja igualmente consenso no que concerne ao valor que corresponde a um bom ajustamento do modelo: enquanto uns referem que deve ser inferior a 2 , outros aceitam que seja tão elevado como 5 (6). A este propósito, Joreskog (21), uma das principais referências neste domínio do conhecimento, sugeriu que valores inferiores a 3 representam uma correspondência aceitável entre o modelo submetido a apreciação e os dados. Assim sendo, temos que tanto o modelo $\mathrm{M}_{12}^{3}$ como o modelo $\mathrm{M}_{24}^{4}$ apresentaram um ajustamento aceitável aos dados analisados, particularmente o primeiro.

Também os valores relativos à RMSEA e à RMRst convergiram no sentido da manifestação da qualidade do modelo $\mathrm{M}^{3}{ }_{12}$, porquanto se situaram perfeitamente dentro dos valores normalmente referidos na literatura da especialidade como os limites a partir dos quais se deve considerar um modelo como aceitável. Aliás, se considerarmos que se aceita que valores de até .08 para a RMSEA representam erros razoáveis de aproximação, apenas sendo de rejeitar valores superiores a $.10(7,8,22)$, verificamos que 
nenhum dos modelos testados se revelou de forma muito negativa neste parâmetro.

Finalmente, conforme é possível verificar através da consulta ao quadro 3 , o cálculo do alfa de Cronbach para cada um dos factores dos quatro modelos investigados revelou que todos apresentaram uma aceitável consistência interna, porquanto nenhum foi inferior ao valor critério de .70 , proposto por Nunnally (28).

Quadro 3. Valores do alfa de Cronbach para todos os factores dos modelos investigados.

\begin{tabular}{lcccc} 
Factores & $\mathrm{M}^{4}{ }_{24}$ & $\mathrm{M}^{3}{ }_{24}$ & $\mathrm{M}^{4}{ }_{16}$ & $\mathrm{M}^{3}{ }_{12}$ \\
\hline Competência Desportiva & .79 & & .74 & \\
Condição Física & .75 & & .74 & \\
Aparência Física & .78 & .78 & .73 & .73 \\
Força Física & .82 & .82 & .79 & .79 \\
Confiança Física & & .86 & & .71
\end{tabular}

\section{DISCUSSÃO E CONCLUSÕES}

$\mathrm{Na}$ origem deste estudo situou-se o reconhecimento de que era necessário investigar, de forma mais profunda e sistemática do que havia sido feito até ao momento, as propriedades psicométricas da versão portuguesa do PSPP, particularmente em relação à sua estrutura factorial e consistência interna. De uma forma geral, as várias análises desenvolvidas ao longo do nosso estudo revelaram que uma estrutura constituída por três factores, e não quatro, se ajustou melhor aos dados examinados. Ou seja, pareceu não haver evidência suficiente para a sustentação da proposta de uma estrutura para o PSPPp similar à identificada para a versão original. Para proceder à elaboração do PSPP, Fox e Corbin $(15,18)$ desenvolveram um extenso trabalho de natureza qualitativa (de entre o qual se pode destacar a realização de entrevistas com indivíduos de diferentes características, análise de conteúdo das suas respostas, revisão da literatura especializada e análise de outros instrumentos já existentes nesta área) e quantitativa (recorrendo a um conjunto bastante alargado e completo de técnicas estatísticas que providenciou um forte suporte para a sua validade e fiabilidade). Nessa medida, não surpreende que tenham sido vários os investigadores que utilizaram o PSPP nas suas investigações e que atestaram a sua validade e fiabilidade para os fins para os quais foi proposto (para mais detalhes, ver [17]).

Tal como referimos anteriormente, e na sequência da importância que lhe é atribuída no domínio da avaliação do modo como as pessoas se percebem fisicamente, o PSPP foi traduzido para diversas línguas e culturas por investigadores que, naturalmente, se preocuparam igualmente em determinar as qualidades psicométricas das versões traduzidas, particularmente no que se refere à sua estrutura factorial. Os resultados não têm sido todavia unânimes; isto é, alguns dos autores que traduziram e adaptaram o PSPP para outras línguas e culturas reportaram dificuldades em identificar a estrutura factorial proposta para a versão original do PSPP.

Por exemplo, Van der Vliet e colaboradores (43), num estudo realizado na Bélgica com a versão flamenga do PSPP para adultos, referiram ter encontrado suporte para uma estrutura constituída por três factores e não quatro (com os factores Competência Desportiva e Condição Física a unirem-se num só factor). Do mesmo modo, Hagger, Ashford e Stambulova $(19,20)$, ao analisarem dados relativos a jovens britânicos e russos, revelaram não ter encontrado evidência suficiente no sentido da confirmação do modelo de quatro factores do PSPP para crianças. Estes autores destacaram ainda que, ao analisarem a invariância estrutural do instrumento (ao longo do género sexual e do país dos participantes no estudo), em todos os conjuntos de dados examinados, os valores de saturação dos diferentes itens nos factores definidos apresentaram um padrão semelhante. Nessa medida, alertaram para a possibilidade de o problema ser de natureza psicométrica, não decorrendo de dificuldades por parte das amostras investigadas, porquanto elas foram consistentes no modo como the responderam.

Importará talvez recordar que a questão do número exacto de factores constituintes do PSPP-C já havia sido colocada, ainda que de forma não tão marcada, em estudos realizados nos Estados Unidos da América por Whitehead e Corbin $(45,46)$. No âmbito destes estudos foram identificados, através do recurso à técnica da $\mathrm{AFE}$, cinco factores e não quatro, bem como verificada a existência de alguns itens com valores de saturação cruzada, particularmente 
nos factores da Aparência Física e da Condição Física, no caso dos rapazes, e dos factores Competência Desportiva e Condição Física, no caso das raparigas.

No mesmo sentido, também Biddle e colaboradores (5), num estudo realizado em Inglaterra, verificaram que as crianças evidenciaram algumas dificuldades em distinguir claramente entre Competência Desportiva e Condição Física, razão pela qual a estrutura de quatro factores proposta para o PSPP-C não foi claramente interpretável a partir da análise das suas respostas.

Num outro estudo desenvolvido em Inglaterra, por Page e colaboradores (31), e não obstante a análise de componentes principais (ACP) às matrizes de correlação dos dados relativos a duas amostras de jovens adultos (idades médias de $19.42 \pm 2.41$ e de $21.13 \pm 6.23$ anos) ter providenciado um forte suporte para a estrutura de quatro factores proposta por Fox (15), foi igualmente evidente que alguns itens, particularmente os relativos à Condição Física, apresentaram valores de saturação cruzada.

Ainda Welk, Corbin e Lewis (44), ao depararem com uma elevada correlação entre as sub-escalas da Condição Física e da Competência Desportiva, bem como com a saturação cruzada de vários itens ao longo das duas sub-escalas, pronunciaram-se acerca da possibilidade de os dois constructos que lhes estão subjacentes não serem entendidos de forma tão distinta pelos inquiridos quanto a que pressupõe a sua separação em duas sub-escalas, sugerindo por isso mesmo a necessidade de mais investigação sobre esta questão. De sublinhar, porém, que uma análise factorial confirmatória posteriormente realizada por Eklund, Whitehead e Welk (11) aos mesmos dados, em combinação com os dados do também já referido estudo de Whitehead (45), providenciou um claro suporte para existência de uma estrutura de quatro factores para o PSPP-C.

Mais recentemente, Asçi, Asçi e Zorba (2), num estudo realizado com a versão turca do PSPP, no qual participaram jovens universitários, identificaram, através do recurso à $\mathrm{ACP}$, uma solução idêntica à da versão original. Do mesmo modo, também Atienza, Balaguer e Moreno (1), num estudo com a versão espanhola do PSPP, realizado com adolescentes, concluíram que os seus resultados providenciaram suporte para a exis- tência de uma estrutura de quatro factores similar à proposta para a versão original do PSPP.

Neste caso, porém, se atentarmos nos resultados da AFC que estiveram na origem daquela conclusão (rapazes: $\chi^{2} / \mathrm{gl}=1.78, \mathrm{RMR}=.06$, AGFI $=.82, \alpha=.76$ .84; e raparigas: $\chi^{2} / \mathrm{gl}=2.42, \mathrm{RMR}=.07, \mathrm{AGFI}=.82$, $\alpha=.79-.85)$ constatamos que eles não foram assim tão inequívocos. Ou seja, apesar dos valores dos outros indicadores poderem ser considerados como aceitáveis, o valor de AGFI não ultrapassou os 0.82 , bastante inferior portanto ao valor de 0.90 sugerido na literatura como o mínimo para se considerar como aceitável o ajustamento do modelo aos dados examinados. Aliás, se atentarmos nos resultados da nossa AFC à estrutura de quatro factores verificamos que não foram assim tão diferentes dos de Atienza e colaboradores (1). Nessa medida, pensamos que subsiste a possibilidade de uma estrutura factorial de três factores, não investigada por aquelas autoras, se ajustar melhor à estrutura de covariância dos seus dados, tal como verificado no nosso estudo.

$\mathrm{Da}$ análise dos resultados dos diversos estudos a que nos referimos anteriormente parece pois resultar que nem sempre tem sido possível identificar uma mesma estrutura factorial para o PSPP, nomeadamente no que se refere às versões que foram entretanto traduzidas para outras línguas e culturas. Ainda assim, pensamos ser de destacar que, de uma forma geral, os resultados dos diferentes estudos nos quais foi utilizado o PSPP têm sido em grande medida consistentes, porquanto para além de em quase todos eles os valores do coeficiente de alfa de Cronbach calculado para os vários factores constituintes do PSPP terem sido superiores ao valor mínimo proposto por Nunnally (28), os principais problemas reportados parecem relacionar-se com a relação mais ou menos pronunciada existente entre os factores Competência Desportiva e Condição Física. Do nosso estudo pareceu emergir igualmente a noção de que os indivíduos que responderam ao PSPPp não efectuaram uma distinção nítida entre aqueles dois conceitos, atendendo a que o ajustamento global do modelo no qual eles foram considerados diferenciadamente foi inferior ao do modelo no qual eles foram entendidos conjuntamente. A este respeito, importará porventura sublinhar que nem em todas as línguas e culturas existe uma dis- 
tinção tão clara entre os conceitos de competência desportiva e condição física, como a habitualmente verificada na língua inglesa, quer a falada nos Estados Unidos da América (onde foi desenvolvido o PSPP) quer a falada no Reino Unido (de onde é originário o seu principal autor), fundamentalmente se consideramos os indivíduos mais velhos e menos associados à pratica de uma actividade física ou desportiva. Isso mesmo foi destacado por Van der Vliet e colaboradores (43), quando verificaram que a partir da análise das respostas dos adultos que participaram no seu estudo não foi possível separar aqueles dois constructos.

Adicionalmente, há que recordar que as idades dos indivíduos que participaram nos diferentes estudos realizados até ao momento neste domínio não foram sempre as mesmas. Na verdade, não são muitos os estudos efectuados com indivíduos adultos, já que a maior parte foi desenvolvida com crianças, adolescentes ou jovens adultos, e o modo como uns e outros se percepcionam em termos físicos parece assumir contornos substancialmente diferenciados. O principal objectivo desta pesquisa consistia na determinação da validade e fiabilidade do PSPPp para avaliar o modo como os adultos se percepcionam fisicamente, tendo os resultados encontrados, no seu conjunto, fornecido suporte suficiente para tal assunção. Ainda assim, consideramos que é necessário desenvolver mais trabalho, por exemplo, no que respeita à análise da sua invariância estrutural ao longo de diferentes amostras para que a sua validade e fiabilidade estejam claramente estabelecidas. Ou seja, entendemos que é importante determinar até que ponto a estrutura factorial agora identificada se revela como igualmente adequada a dados provenientes de amostras distintas da por nós investigada (e.g., indivíduos não praticantes, idosos, jovens, com necessidades educativas especiais). Inclusivamente, é importante recordar que mais de um modelo pode ajustar-se de forma aceitável a um conjunto de dados (4), o que significa que o facto do modelo de medida por nós inspeccionado ter evidenciado uma boa qualidade global de ajustamento aos dados não equivale a dizer que não pode haver um outro modelo a apresentar ainda melhores resultados, razão adicional, portanto, para serem reclama- dos mais esforços no sentido da definição da melhor estrutura factorial para o PSPPp.

Finalmente, de destacar que Page e colaboradores

(31) alertaram para a necessidade de investigar a sensibilidade do PSPP para avaliar alterações decorrentes da prática de actividade física, ou da participação em programas de controlo do peso ou de outro tipo, propósito para o qual o PSPP tem efectivamente vindo a ser utilizado (10). Nessa medida, entendemos que seria igualmente importante equacionar a possibilidade de futuramente ser desenvolvida uma escala destinada a avaliar o que poderíamos designar de capacidade física funcional percebida pelos indivíduos, porquanto em estudos com adultos, particularmente os mais idosos, esse é um aspecto a ter em atenção. 


\section{REFERÊNCIAS}

1. Atienza, F., Balaguer, I. \& Moreno, Y (1997). Factorial validity of the Physical Self-Perception Profile (PSPP) in the case of Spanish adolescents. In R. Lidor \& M. Bar-Eli (Eds), Innovations in sport psychology: Linking theory and practice (vol.1, pp.82-84). Netanya, Israel: The Zinman College of Physical Education and Sport Sciences \& The Wingate Institute for Physical Education and Sport.

2. Asçi, F. H., Asçi, A. \& Zorba, E. (1999). Cross-cultural validity and reliability of Physical Self-Perception Profile. International Journal of Sport Psychology, 30, 399-406.

3. Bentler, P. M. (1990). Comparative fit indexes in structural models. Psychological Bulletin, 107 (2), 238-246.

4. Biddle, B.J. \& Martin, M.M. (1987). Causality, confirmation, credulity, and structural equation modelling. Child Development, 58, 4-17.

5. Biddle, S., Page, A., Ashford, B., Jennings, D., Brooke, R. \& Fox, K. (1993). Assessment of children's physical selfperceptions. International Journal of Adolescence and Youth, 4, 93-109.

6. Bollen, K. A. (1989). Structural equations with latent variables. New York: John Wiley \& Sons, Inc.

7. Browne, M.W. \& Cudeck, R. (1993). Alternative ways of assessing model fit. In Kenneth A. Bollen \& J. Scott Long (Eds.), Testing structural equation models (pp.136-162). Newbury Park, CA: Sage Publications.

8. Byrne, B. M. (1998). Structural equation modeling with LISREL, PRELIS, and SIMPLIS: Basic concepts, applications and programming. Mahwah, NJ: Lawrence Erlbaum Associates.

9. Cruz, J.F., Machado, P.P., \& Mota, M.P. (1996). Efeitos e benefícios psicológicos do exercício e da actividade física. In José F. Cruz (Ed.), Manual de psicologia do desporto (pp.91-116). Braga: SHO-Sistemas Humanos e Organizacionais.

10. Daley, A. J. \& Buchanan, J. (1999). Aerobic dance and physical self-perceptions in female adolescents: Some implications for Physical Education. Research Quarterly for Exercise and Sport, 70(2), 196-200.

11. Eklund, R.C., Whitehead, J.R. \& Welk, G.J. (1997). Validity of the Chlidren and Youth Physical Self-Perception Profile: A confirmatory factor analysis. Research Quarterly for Exercise and Sport, 68(3), 249-256.

12. Fonseca, A.M., Fox, K., \& Almeida, M.J. (1995). Versão portuguesa do Physical Self-Perception Profile (PSPP): O Perfil de Auto-Percepção Física (PSPPp). Trabalho não publicado. FCDEF, Universidade do Porto.

13. Fonseca, A.M., Fox, K.R. \& Almeida, M.J. (1997). Projecto de Investigação e Promoção da Actividade Física das Populações (PIPAFP). Comunicação apresentada no I Fórum das Universidades que integram o Convénio das Licenciaturas em Ciências do Desporto. Vila Real: UTAD.

14. Fonseca, A.M., Lago, J. \& Mota, J. (1997). Estudo inicial sobre as relações entre a assiduidade e as autopercepções físicas e de coesão de grupo em classes de ginástica de academia. Actas da $V$ Conferência Internacional "Avaliação Psicológica: Formas e contextos" \& III Mostra de "Provas Psicológicas em Portugal” (pp.450-460). Braga: APPORT \& Universidade do Minho.

15. Fox, K. R. (1990). The Physical Self-Perception Profile manual. DeKalb, IL: Office for Health Promotion, Northern Illinois University.
16. Fox, K. (Ed.) (1997). The physical self: From motivation to well-being. Champaign, IL: Human Kinetics.

17. Fox, K. R. (1998). Advances in the measurement of the physical self. In Joan L. Duda (Ed.), Advances in sport and exercise psychology measurement (pp.295-310). Morgantown, WV: Fitness Information Technology.

18. Fox, K. R. \& Corbin, C. B. (1989). The Physical SelfPerception Profile: Development and preliminary validation. Journal of Sport and Exercise Psychology, 11(4), 408-430.

19. Hagger, M., Ashford, B. \& Stambulova, N. (1997). Physical self-perceptions: A cross-cultural assessment in Russian children. European Journal of Physical Education, 2(2), 228-245.

20. Hagger, M., Ashford, B. \& Stambulova, N. (1998). Russian and British children's physical self-perceptions and physical activity partiicpation. Pediatric Exercise Science, 10, 137-152.

21. Joreskok, K.J. (1969). A general approach to confirmatory maximum likehood factor analysis. Psychometrica, 34, 183-202.

22. MacCallum, R.C., Browne, M.W., \& Sugawara, H.M. (1996). Power analysis and determination of sample size for covariance structure modeling. Psychological Methods, 1 130-149.

23. Marsh, H. W., (1997). The measurement of physical selfconcept: A construct validation approach. In K.R.Fox (Ed), The physical self: From motivation to well-being (pp.27-58). Champaign, IL: Human Kinetics.

24. Marsh, H.W., Balla, J.R. \& McDonald, R.P. (1988). Goodness-of-fit indexes in confirmatory factor analysis: The effect of sample size. Psychological Bulletin, 103 (3), 391-410.

25. Marsh, H. W., Richards, G. E., Johnson, S., Roche, L., \& Tremayne, P. (1994). Physical Self-Description Questionnaire: Psychometric properties and a multitraitmultimethod analysis of relations to existing instruments. Journal of Sport and Exercise Psychology, 16(3), 270-305.

26. McDonald, R.P. \& Marsh, H.W. (1990). Choosing a multivariate model: Noncentrality and goodness-of-fit. Psychological Bulletin, 107, 247-255.

27. Mulaik, S. A. (1987). A brief history of the philosophical foundations of exploratory factor analysis. Multivariate Behavioral Research, 22, 267-305.

28. Nunnally, J.C. (1978). Psychometric theory ( $2^{a}$ edição). St.Louis: McGraw-Hill.

29. Oliveira, J. \& Fonseca, A.M. (2000a). Autopercepções físicas, motivação intrínseca e intenção de praticar natação de lazer. Comunicação apresentada no XXIV Congresso TécnicoCientífico da APTN. Rio Maior.

30. Oliveira, J. \& Fonseca, A.M. (2000b). Relações entre autopercepções físicas, motivação intrínseca e assiduidade em classes de natação de lazer. Comunicação apresentada no XXIV Congresso Técnico-Científico da APTN. Rio Maior.

31. Page, A., Ashford, B., Fox, K., \& Biddle, S. (1993). Evidence of cross-cultural validity for the Physical SelfPerception Profile. Personality and Individual Differences, 14(4), 585-590.

32. Pedhazur, E.J. \& Schmelkin, L.P. (1991). Measurement, design, and analysis: An integrated approach. Hillsdale, NJ: Lawrence Erlbaum Associates.

33. Rosenberg, M. (1989). Society and the adolescent self-image (ed. rev.). Middletown, $\mathrm{CN}$ : Wesleyan University Press.

34. Sallis, J. \& Owen, N. (1999). Physical Activity \& Behavioral Medicine. Newbury Park, CA: Sage Publications. 
35. Sonstroem, R.J. (1984). Exercise and self-esteem. Exercise and Sports Sciences Reviews, 12, 123-155.

36. Sonstroem, R.J. (1997). The physical self-system: A mediator of exercise and self-esteem. In K.R.Fox (Ed), The physical self: From motivation to well-being (pp.3-26). Champaign, IL: Human Kinetics

37. Sonstroem, R., Harlow, L.L. \& Josephs, L. (1994) Exercise and self-esteem: validity of model expansion and exercise associations. Journal of Sport and Exercise Psychology, 16(1), 29-42.

38. Sonstroem, R.J. \& Morgan, W.P. (1989). Exercise and selfesteem: Rationale and model. Medicine and Science in Sports and Exercise, 28, 619-625.

39. Sonstroem, R. J., Speliotis, E. D., \& Fava, J. L. (1992). Perceived physical competence in adults: An examination of the Physical Self-Perception Profile. Journal of Sport and Exercise Psychology, 14(2), 207-221.

40. Stevens, J. (1996). Applied multivariate statistics for the social sciences (3rd edition). Mahwah, NJ : Lawrence Erlbaum Associates.

41. Tabachnick, B.G. \& Fidell, L.S. (1997). Using multivariate statistics (3rd edition). New York: Harper Collins.

42. Vallerand, R.J. (1989). Vers une méthodologie de validation transculturelle de questionnaires psychologiques: Implications pour la recherche en langue française.

Canadian Psychology/Psychologie Canadienne, 30(4), 662-680.

43. Van de Vliet, P., Knapen, J., Onghena, P., Fox, K., Van Coppenolle, H., David, A. Pieters, G., \& Peuskens, J. (no prelo). Assessment of physical sel-perceptions in normal Flemish adults versus depressed psychiatric patients.

44. Welk, G. J., Corbin, C. B., \& Lewis, L. A. (1995). Physical self-perceptions of high school athletes. Pediatric Exercise Science, 7(2), 152-161.

45. Whitehead, J.R. (1995). A study of children's physical selfperceptions using an adapted Physical Self-Perception

Profile questionnaire. Pediatric Exercise Science, 7(2), 132-151.

46. Whitehead, J.R. \& Corbin, C. (1988). Physical

competence/adequacy subdomains and their relationship to global physical self-worth and global general self-worth. Comunicação apresentada na Conferência anual da Association for the Advancement of Applied Sports Psychology (AAASP).

Nashua, NH. 